\title{
Efectos Dinámicos de la Política Fiscal*
}

\author{
Rodrigo A. CERDA \\ Hermann GonZÁlez \\ Luis Felipe Lagos** \\ Pontificia Universidad Católica de Chile
}

This paper identifies the dynamic effects of fiscal policy on economic activity $(G D P)$ in the Chilean economy. We use a structural vector autoregression (SVAR) methodology. The main results are: a positive fiscal expenditure shock has a negative effect on output during the first quarter; afterwards the effect dies out. A positive tax shock also has a negative marginal impact on output for one quarter.

JEL: H20, H30

Keywords: Fiscal Expenditure Shock, SVAR

\section{INTRODUCCIÓN}

Investigaciones recientes (Perotti 2002, por ejemplo) han concluido que los efectos expansivos de la política fiscal sobre el producto de países desarrollados se han debilitado en los últimos 20 años. Incluso se reporta un efecto negativo del gasto fiscal sobre el producto para países de la OECD, lo que contradice la visión keynesiana tradicional de la política fiscal como herramienta anticíclica.

Sorprendentemente, y a diferencia de la política monetaria, existe poca evidencia respecto de los efectos de la política fiscal para el caso de Chile. La escasa evidencia empírica podría explicarse por el acceso relativamente más difícil a los datos de gasto e impuestos.

Este trabajo busca reportar evidencia respecto de los efectos de la política fiscal sobre la actividad económica. Se estudian los efectos dinámicos del gasto fiscal e impuestos sobre el producto para la economía chilena usando la metodo-

\footnotetext{
* Se agradecen los comentarios de participantes en el seminario interno de la Pontificia Universidad Católica de Chile, así como los comentarios de un arbitro anónimo, que sirvieron para mejorar sustancialmente este trabajo.

***E-mails: rcerda@faceapuc.cl, flagos@faceapuc.cl, hgonzalb@bcentral.cl
} 
logía de vectores autorregresivos estructurales. Los resultados indican que un shock positivo de gasto fiscal tiene un efecto negativo y significativo sobre el producto sólo durante el primer trimestre; mientras que un shock positivo de impuesto tiene un efecto impacto negativo, pero de muy baja magnitud y sólo durante un trimestre, sobre el producto. El trabajo posteriormente entrega algunas ideas que podrían servir para explicar estos efectos. Sin embargo, estos planteamientos son simples hipótesis, dado que no se presenta evidencia de ellos.

El trabajo se organiza de la siguiente forma: la sección 2 revisa los resultados de la literatura que usan modelos VAR; la sección 3 desarrolla la metodología empírica del trabajo y en la sección 4 se presentan los resultados. Posteriormente, en la sección 5 se entregan posibles explicaciones a los resultados reportados y se concluye.

\section{El Uso de VAR Estructurales Para El Análisis De La Política FisCAL}

La gran mayoría de los estudios recientes relativos a los efectos de la política fiscal usan como herramienta econométrica modelos de vectores autorregresivos (VAR). ${ }^{1}$ Cabe destacar que la mayor parte de los estudios acerca de la efectividad de la política fiscal se basan en la experiencia de países desarrollados, debido principalmente a la disponibilidad de información.

Blanchard y Perotti (1999) analizan mediante la metodología de VAR estructurales los efectos dinámicos de shocks de gasto de gobierno e impuestos en la actividad económica de Estados Unidos y encuentran que los shocks positivos de gasto fiscal tienen un efecto positivo en el producto; la duración de este shock es mayor que la de losshocks de impuestos y alcanza su máximo efecto después de casi cuatro años; los impuestos netos también responden de forma positiva, probablemente como consecuencia de la respuesta del PIB (se puede notar que la evolución de las funciones de impulso respuesta de impuesto y PIB son similares). Los shocks positivos de impuestos tienen efectos negativos y significativos en el PIB, produciéndose el mayor efecto después de cinco a siete trimestres. Además, al analizar los efectos sobre los componentes del PIB encuentran que tanto un aumento del gasto de gobierno como de los impuestos tienen un fuerte efecto negativo sobre la inversión.

Perotti (2002) extiende el trabajo de 1999 y estima un modelo VAR estructural con datos trimestrales para cinco países: Australia, Canadá, Alemania, Reino Unido y Estados Unidos. Los resultados muestran un impacto positivo y significativo del gasto de gobierno en el producto en toda la muestra. Después del aumento inicial, el PIB comienza a declinar y luego aumenta de nuevo, aunque sólo en Alemania y Estados Unidos este aumento es estadísticamente significativo. De hecho, durante los tres primeros años ocurre el mayor impacto en todos los países.

\footnotetext{
${ }^{1}$ Blanchard y Perotti (1999), Perotti (2002), Fatás y Mihov (2001), van Aarle et al. (2001).
} 
Estos resultados difieren si se estima el modelo separando la muestra antes y después de 1980, encontrándose que en todos los países, excepto Australia, los efectos del gasto de gobierno en el PIB son substancialmente inferiores en el período posterior a 1980. De hecho, en este caso, la respuesta instantánea del PIB en cuatro países nunca es estadísticamente significativa y es negativa en los tres primeros años. La excepción es Australia, donde el efecto es positivo y significativo en el período post 1980 y cero en el período anterior.

Sólo en Estados Unidos y Canadá el efecto impacto de los impuestos en el producto es negativo, aunque muy pequeño, cuando se considera toda la muestra. En los demás países el efecto impacto es positivo y significativo, y después de unos trimestres, la respuesta es negativa en todos los países en el período pre 1980 (aunque no significativa en Australia). En el período post 1980, el efecto impacto es positivo en todos los países excepto en Canadá y la evolución futura llega a ser significativamente negativa sólo en Estados Unidos.

Nuestro estudio buscará extender el análisis para el caso de Chile, con la finalidad de estudiar un país en desarrollo. Nos centraremos en el caso chileno porque Chile ha presentado algunos episodios de ajustes fiscales interesantes en los últimos 20 años, como, por ejemplo, un importante ajuste fiscal a mediados de los ochenta, y su posterior reversión al realizarse el cambio de gobierno de 1990. Este escenario nos permite tener un experimento adecuado para evaluar el efecto de la política fiscal. En segundo lugar, el gobierno de Chile publica datos de ejecución del presupuesto fiscal con frecuencia trimestral desde 1986. Este segundo requisito nos permite trabajar con datos de alta frecuencia. Esta es una propiedad de los datos, tal como se explicará más abajo, que nos facilitará identificar los shocks fiscales.

\section{Metodología De Estimación De Los Efectos Dinámicos De La Política Fiscal En El PIB}

La idea del trabajo es identificar shocks de política fiscal y a partir de ellos obtener estimaciones de los efectos de cambios fiscales exógenos sobre los niveles de actividad económica. Con esa finalidad, este estudio trabajará con VAR estructurales con datos de alta frecuencia. Las razones para ocupar esta metodología son de dos tipos. La primera de ellas es que creemos posible identificar los shocks fiscales. De hecho, las variables determinadas por el fisco se mueven por muchas causas, entre las cuales la estabilización del producto es la que menos parece predominar. ${ }^{2}$ Desde este punto de vista pareciera que existen shocks fiscales exógenos respecto al movimiento en el producto. Si es posible identificar los

\footnotetext{
${ }^{2} \mathrm{El}$ balance fiscal responde a decisiones discrecionales de política y a factores exógenos, como inercia de ciertos gastos o compromisos de arrastre de inversiones de períodos anteriores, y a factores cíclicos atribuibles a la actividad económica y precio del cobre. Véase Marcel et al. (2001).
} 
shocks, se puede ocupar la metodología impulso-respuesta de los VAR para identificar sus efectos sobre la actividad económica. En segundo lugar, los rezagos con que opera la política fiscal permiten que al analizar datos de alta frecuencia haya una respuesta débil o nula de la política fiscal corriente ante movimientos inesperados de la actividad económica, lo que nos permitirá más fácilmente identificar los shocks fiscales.

El principal argumento en contra de la metodología es que supone que los shocks de política fiscal no son anticipadas por el sector privado. Este es un supuesto que podría no ser realista, en la medida que la mayoría de las modificaciones en el gasto fiscal e impuestos son conocidas por el público algunos trimestres antes de ser implementadas.

En este trabajo se utilizará un modelo VAR estructural similar al propuesto por Blanchard y Perotti (1999) y utilizado por Perotti (2002) para determinar los efectos de la política fiscal (gasto e impuestos) sobre el PIB con datos para Chile en el período 1986 - 2001. La contribución de la metodología econométrica es que busca identificar los movimientos exógenos versus los movimientos endógenos de la política impositiva y la política de gasto fiscal. De esta forma, una vez identificados los shocks exógenos en la política fiscal, se busca determinar el efecto de estos cambios exógenos de política fiscal sobre la actividad económica.

\subsection{Descripción de Datos}

A continuación, se explica el procedimiento econométrico, deteniéndonos principalmente en (1) la metodología de identificación de los shocks exógenos de política fiscal, y en (2) la metodología que nos permite obtener las funciones de respuesta de nuestras variables endógenas (especialmente la actividad económica) y sus intervalos de confianza ante un shock de política fiscal.

El modelo considera las siguientes variables: gasto de gobierno $G_{t}$, impuestos netos de subsidios $T_{t}$, y producto $Y_{t}$ donde todas estas variables están medidas en millones de pesos de 1996, en logaritmo y ajustadas estacionalmente. La fuente de estos datos es Correa et al. (2003). Las variables de política fiscal se tomaron de datos mensuales publicados en el Informe Financiero del Tesoro Público de la Tesorería General de la República. La serie de recaudación tributaria corresponde a la suma del impuesto a la renta, IVA, a impuestos al comercio exterior, a impuestos a productos específicos, a los actos jurídicos y otros impuestos netos de subsidios. El gasto de gobierno corresponde al gasto total del Tesoro Público que incluye transferencias, prestaciones previsionales, inversión financiera, servicio de la deuda pública y aporte fiscal libre.

El Cuadro 1 presenta test de raíces unitarias para las tres series. Todas las series están desestacionalizadas utilizando el método X12-Arima. Para elegir los rezagos en los test ADF y DF-GLS, se utiliza el criterio de Akaike. En el caso de los test en niveles se incluye constante y tendencia en las tres series, y en el de primeras diferencias se incluye constante. En general, las series resultan ser no estacionarias en niveles, pero estacionarias en sus primeras diferencias. 
CUADRO 1

TEST DE RAICES UNITARIAS, 1986.1- 2004:2

\begin{tabular}{|c|c|c|c|c|c|c|c|c|c|}
\hline & $\begin{array}{l}\text { Valor } \\
\text { Crítico }\end{array}$ & $\begin{array}{l}\text { Valor } \\
\text { Test }\end{array}$ & Serie & $\begin{array}{l}\text { Valor } \\
\text { Crítico }\end{array}$ & $\begin{array}{l}\text { Valor } \\
\text { Test }\end{array}$ & Serie & $\begin{array}{l}\text { Valor } \\
\text { Crítico }\end{array}$ & $\begin{array}{l}\text { Valor } \\
\text { Test }\end{array}$ & Serie \\
\hline & \multicolumn{3}{|c|}{ Log PIB } & \multicolumn{3}{|c|}{ Log Gasto } & \multicolumn{3}{|c|}{$\log \operatorname{Tax}$} \\
\hline ADF & $1 \%,-4.11$ & -0.85 & $\mathrm{I}(1)$ & $1 \%,-4.11$ & -1.20 & I(1) & $1 \%,-4.08$ & -2.88 & I(1) \\
\hline PP & $\begin{array}{l}5 \%,-3.48 \\
1 \%,-4.11\end{array}$ & -0.95 & $\mathrm{I}(1)$ & $\begin{array}{l}5 \%,-3.48 \\
1 \%,-4.11\end{array}$ & -1.67 & $\mathrm{I}(1)$ & $\begin{array}{l}5 \%,-3.47 \\
1 \%,-4.08\end{array}$ & -2.75 & $\mathrm{I}(1)$ \\
\hline \multirow{4}{*}{$\begin{array}{l}\text { (Philips Perron) } \\
\text { DF-GLS } \\
\text { (Dickey - Fuller } \\
\text { GLS) }\end{array}$} & $5 \%,-3.48$ & & & $5 \%,-3.48$ & & & $5 \%,-3.47$ & & \\
\hline & $1 \%,-3.72$ & -0.58 & $\mathrm{I}(1)$ & $1 \%,-3.72$ & 0.92 & $\mathrm{I}(1)$ & $1 \%,-3.68$ & -2.90 & $\mathrm{I}(1)$ \\
\hline & $5 \%,-3.15$ & & & $5 \%,-3.15$ & & & $5 \%,-3.11$ & & \\
\hline & \multicolumn{3}{|c|}{$\mathrm{D}(\ln (\mathrm{PIB}))$} & \multicolumn{3}{|c|}{$\mathrm{D}(\ln ($ Gasto $))$} & \multicolumn{3}{|c|}{$\mathrm{D}(\ln (\operatorname{Tax}))$} \\
\hline ADF & $\begin{array}{l}1 \%,-3.54 \\
5 \%,-2.90\end{array}$ & -6.46 & $\mathrm{I}(0)$ & $\begin{array}{l}1 \%,-3.54 \\
5 \%,-2.90\end{array}$ & -7.81 & $\mathrm{I}(0)$ & $\begin{array}{l}1 \%,-3.52 \\
5 \%,-2.90\end{array}$ & -10.1 & $\mathrm{I}(0)$ \\
\hline PP & $1 \%,-3.54$ & -6.49 & $\mathrm{I}(0)$ & $1 \%,-3.54$ & -12.3 & $\mathrm{I}(0)$ & $1 \%,-3.52$ & -10.8 & $\mathrm{I}(0)$ \\
\hline (Philips Perron) & $5 \%,-2.90$ & & & $5 \%,-2.90$ & & & $5 \%,-2.90$ & & \\
\hline DF-GLS & $1 \%,-2.60$ & -2.51 & $\mathrm{I}(0)$ & $1 \%,-2.60$ & -4.92 & $\mathrm{I}(0)$ & $1 \%,-2.59$ & -9.02 & $\mathrm{I}(0)$ \\
\hline (Dickey - Fuller GLS) & $5 \%,-1.94$ & & & $5 \%,-1.94$ & & & $5 \%,-1.94$ & & \\
\hline
\end{tabular}

Todas las variables están desestacionalizadas por medio del método X12Arima. Los test concernientes a las variables en niveles incluyen constante y tendencia, mientras que los test de las variables en primeras diferencias incluyen sólo constante.

\subsection{El Sistema VAR}

Como las series estacionarias son las series en primeras diferencias, se decidió realizar estimaciones utilizando un VAR estructural ocupando estas últimas variables. Sea $X_{t}$ el vector de variables endógenas. Entonces se escribe la siguiente forma reducida para el sistema VAR:

(1) $\quad X_{t}=\sum_{j=1}^{K} A_{t-j} X_{t-j}+U_{t}$

Donde $X_{t} \equiv\left[\Delta G_{t} \Delta Y_{t} \Delta T_{t}\right]^{\prime}$ y $U_{t}$ es un vector de residuos definido como $U_{t} \equiv\left[t_{t} g_{t} y_{t}\right]^{\prime}$. El símbolo $\Delta$ indica primeras diferencias. El número de rezagos, $\mathrm{K}$, se determinó de acuerdo al criterio de Akaike y se fijó en 8 rezagos. Los residuos juegan un rol bastante importante en el ejercicio realizado en este trabajo. De hecho, en nuestro ejercicio buscamos identificar cambios no anticipados en las decisiones de política económica que forman parte de $U_{t}$. Sin embargo, existen claras presunciones de que el vector de residuos $U_{t}$ contiene información adicional a los cambios exógenos de política fiscal. Es bastante factible que existan errores de medición en nuestras variables que se manifiesten en los residuos, distintas a los shocks de política fiscal, que también afecten al vector de residuos. Por lo tanto, a continuación trataremos de separar los movimientos en los residuos que estén relacionados con los shocks exógenos de política fiscal de otro tipo de efectos. La notación que ocuparemos para identificar los shocks exógenos que se quieren recuperar es $\left\{e_{t}^{t}, e_{t}^{g}, e_{t}^{y}\right\}$ que representan los movimientos inesperados y 
exógenos de impuestos, gasto de gobierno y producto respectivamente. Por lo tanto, para la discusión posterior será importante distinguir entre errores, $\left(t_{t} g_{t} y_{t}\right)$, y shocks exógenos $\left(e_{t}^{t} e_{t}^{g} e_{t}^{y}\right)$. Estos últimos tienen que ver con cambios exógenos que afecten el nivel de actividad y a la política fiscal, mientras que los primeros son funciones de los shocks exógenos.

Para realizar el procedimiento de identificación de los shocks de política fiscal, supondremos que los movimientos inesperados de impuestos -es decir, los residuos de la ecuación de impuestos, $t_{t}-$ se pueden deber a tres factores:

1. La respuesta automática a movimientos inesperados la economía, capturados por los residuos de la ecuación del PIB.

2. La respuesta a shocks inesperados y exógenos de gasto de gobierno, capturados en $e_{t}^{g}$.

3. Shocks inesperados y exógenos de impuestos, capturados en $e_{t}^{t}$.

De esta forma, supondremos una descomposición lineal como la siguiente:

$$
t_{t}=a_{1} y_{1}+a_{2} e_{t}^{g}+e_{t}^{t}
$$

El primero de estos factores capta los componentes de variables omitidas que afecten al nivel general de actividad y, por lo tanto, impacten la evolución de la recaudación de impuestos. Los otros factores captan los cambios exógenos en la política fiscal. La inclusión del shock exógeno de impuestos en $\mathrm{t}_{\mathrm{t}}$ resulta bastante obvio, dado que, por ejemplo, un aumento no anticipado en la tasa de impuestos debería afectar la recaudación de impuestos corrientes, siendo este efecto no captado por la evolución de la actividad económica o rezagos de la misma recaudación de impuestos o gasto fiscal.

Una interpretación similar se aplica a los movimientos inesperados en el gasto. La ecuación (3) describe la descomposición del residuo de la ecuación de gasto de gobierno. Finalmente, supondremos que los movimientos inesperados en el producto se pueden deber a movimientos inesperados en los impuestos, en el gasto o a otros shocks inesperados, $e_{t}^{y}$ (ecuación 4). En relación a la racionalidad de la ecuación que descompone los residuos del PIB es importante indicar que el componente $e_{t}^{y}$ se relaciona con shocks exógenos al nivel de actividad, mientras que la inclusión de los movimientos inesperados en impuestos y gastos tienen que ver con los impactos de la política fiscal sobre el nivel de actividad de la economía. De esta forma, las ecuaciones son:

$$
\begin{aligned}
& g_{t}=b_{1} y_{1}+b_{2} e_{t}^{t}+e_{t}^{g} \\
& y_{t}=c_{1} t_{1}+c_{2} g_{t}+e_{t}^{y}
\end{aligned}
$$




\subsection{Identificación de los Shocks Exógenos}

A continuación se describe la metodología utilizada para identificar los $s h o c k s$ exógenos de política fiscal $e_{t} t$ y $e_{t} g$. Lo primero que debemos notar es que los coeficientes $a_{1}$ y $b_{1}$ pueden capturar dos tipos de efectos de la actividad económica en los impuestos y gasto de gobierno: (i) los efectos automáticos de la actividad económica y (ii) cualquier ajuste discrecional hecho a la política fiscal en respuesta a eventos inesperados durante un trimestre. Un elemento clave del proceso de identificación es el hecho de que el uso de datos trimestrales podría eliminar el segundo canal. Como se sabe, la política fiscal tiene importantes rezagos de diseño, aprobación e implementación, entonces es probable que el proceso de reacción de la política fiscal ante un shock de producto tome más de un trimestre.

Ahora, el problema econométrico que principalmente enfrentamos es que el sistema de ecuaciones (2)-(4) es simultáneo y se requiere algún conjunto de instrumentos que nos permitan identificar los cambios exógenos en el nivel de actividad. Como es posible que el efecto (ii) no esté presente al utilizar datos trimestrales, nuestro instrumento buscará estar relacionado con (i), pero no correlacionado con shocks exógenos de política fiscal (sean de impuestos o gasto). El instrumento que utilizaremos en ese contexto son las variaciones de términos de intercambio, que deben tener un impacto directo sobre producto, y además es una variable determinada por el entorno extorno y por lo tanto, no depende de la política fiscal.

Para estimar otros parámetros del sistema (2)-(4), necesitamos otros tipos de instrumentos. Para identificar el parámetro relacionado con $e_{t}^{t}$ se utiliza el momento de las reformas impositivas ocurridas al comienzo de los noventas. Efectivamente, estas reformas deben producir shocks de recaudación tributaria que impacten $e_{t}^{t}$, pero además estas reformas fueron el fruto de cambios de gobierno, que, por lo tanto, no están relacionados con shocks exógenos de producto. Específicamente ocuparemos cuatro variables mudas, cuya particularidad es que la primera variable muda es igual a uno en el primer trimestre de 1990 y cero en el resto de los datos, mientras que la segunda variable muda es igual a uno en el segundo trimestre de 1990 y cero en el resto. La tercera y cuarta variable muda siguen similar comportamiento, pero son iguales a uno en el tercer y cuarto trimestre de 1990, respectivamente.

Con estas ideas en mente, para identificar los parámetros de las ecuaciones (2)-(4) se siguen los siguientes pasos:

1. Para obtener estimadores consistentes de $a_{1}$ o $b_{1}$, se ocupa 2SLS en (2) y (3) utilizando variaciones en términos de intercambios, como instrumento de $\mathrm{y}_{t}$. Estos estimadores son consistentes y se denotan como $\hat{a}_{1}, \hat{b}_{1}$.

2. Una vez obtenidos $\hat{a}_{1}, \hat{b}_{1}$, se define $\tilde{t}_{t}=t_{t}-\hat{a}_{1} y_{t}, \tilde{g}_{t}=g_{t}-\tilde{b}_{1} y_{t}$. A partir de estas definiciones y de las ecuaciones (2) y (3), se obtiene la siguiente ecuación: $\tilde{g}_{t}=b_{2} \tilde{t}_{t}+e_{t}^{g}\left(1-a_{2} b_{2}\right)$, Como $\tilde{t}_{t}$, $\tilde{g}_{t}$ son variables conocidas 
de nuestra construcción anterior, procedemos a identificar $\hat{b}_{2}$ por medio de 2SLS en $\tilde{g}_{t}=b_{2} \tilde{t}_{t}+e_{t}^{g}\left(1-a_{2} b_{2}\right)$, utilizando como instrumento para $\tilde{t}_{t}$ e 1 momento de las reformas impositivas ocurridas en Chile. Específicamente, se ocupan cuatro variables dummies que son iguales para cada trimestre de 1990 .

3. Utilizando la estimación de $b_{2}$ se puede obtener una proxy de $e_{t}^{g}$, de la siguiente forma: $\tilde{g}_{t}-b_{2} \tilde{t}_{t}=e_{t}^{g}\left(1-a_{2} b_{2}\right)$. Esta variable proxy del residuo se denota $\hat{e}_{t}^{g}$,y se ocupa en la ecuación (2), que se rescribe como $\tilde{t}_{t}=t_{t}-\hat{a}_{1} y_{t}=a_{2} \hat{e}_{t}^{g}+e_{t}^{t}$. Dado que $e_{t}^{g}$ y $e_{t}^{t}$ son shocks exógenos, y por lo tanto ortogonales entre sí, se corre esta ecuación por OLS para obtener las estimaciones $\left(\hat{a}_{2}, \hat{e}_{t}^{t}\right)$.

4. Finalmente para identificar $\left(c_{1}, c_{2}\right)$, se corre la ecuación (4) por 2SLS utilizando $\left(\hat{e}_{t}^{g}, \hat{e}_{t}^{t}\right)$ como instrumentos para $\left(e_{t}^{g}, e_{t}\right)$.

La estimación de este procedimiento entrega los siguientes resultados:

CUADRO 2

ESTIMACION DE LOS PARAMETROS $\left(\mathrm{a}_{1}, \mathrm{~b}_{1}, \mathrm{c}_{1}, \mathrm{a}_{2}, \mathrm{~b}_{2}, \mathrm{c}_{2}\right)$

\begin{tabular}{ccccccc}
\hline Parámetro & $\mathrm{a}_{1}$ & $\mathrm{a}_{2}$ & $\mathrm{~b}_{1}$ & $\mathrm{~b}_{2}$ & $\mathrm{c}_{1}$ & $\mathrm{c}_{2}$ \\
\hline Valor & 1.31 & 0.33 & $1.92 * *$ & -0.05 & -0.03 & $-0.25^{* *}$ \\
(Desviación estándar) & $(0.76)$ & $(0.21)$ & $(0.49)$ & $(0.09)$ & $(-0.03)$ & $(0.05)$ \\
\hline
\end{tabular}

** Indica parámetro significativo al $1 \%$.

Finalmente, nótese que el conjunto de ecuaciones (2)-(4), utilizando esta información, puede volver a escribirse de forma reducida como:

$$
U_{t}=\left[\begin{array}{l}
t_{t} \\
g_{t} \\
y_{t}
\end{array}\right]_{t}=C\left[\begin{array}{c}
e_{t}^{t} \\
e_{t}^{g} \\
e_{t}^{y}
\end{array}\right]
$$

donde

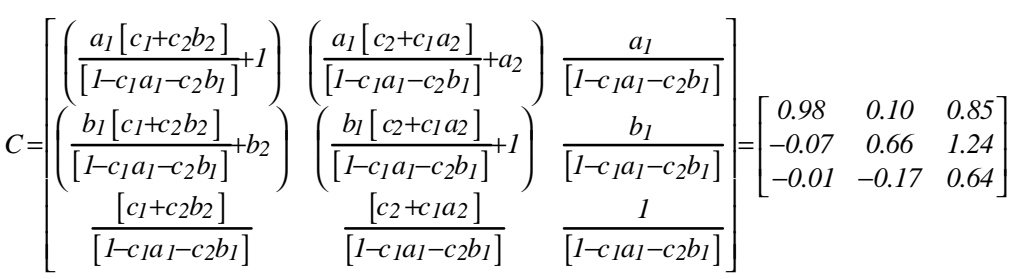

Uno de los resultados que puede parecer interesante de la estimación de los parámetros, dada la estructura de la matriz C, es que el impacto de un shock 
exógeno de $1 \%$ de gasto fiscal tiene un impacto corriente negativo e igual a $-0.17 \%$ del PIB, mientras que el shock exógeno de $1 \%$ en impuestos produce una caída instantáneo de $-0.01 \%$ del PIB (resultados de la última fila de la matriz C). Por otro lado los shocks de impuestos y producto se traspasan instantáneamente de forma casi total a la recaudación fiscal (primera fila de C).

\subsection{Funciones Impulso-Respuesta e intervalos de confianza}

Una particularidad que distingue al VAR recién identificado con los VAR usualmente estimados en la literatura es que la matriz $\mathrm{C}$ no es una matriz identidad. Esta particularidad es muy interesante porque indica que, por ejemplo, un shock de gasto fiscal tiene efectos inmediatos no sólo en la ecuación de gasto sino que también influye directamente sobre la recaudación tributaria, tal como lo indica nuestra estimación de C. De esta forma, las funciones impulso-respuesta entregadas por los paquetes estadísticos tradicionales, al asumir que los shocks no se propagan a través del sistema de ecuaciones, no entregan el verdadero recorrido de las variables ante un shock de gasto o de producto.

Para obtener las "verdaderas" funciones impulso-respuesta seguiremos el siguiente procedimiento. Primero escribimos el VAR de la siguiente forma:

$$
\begin{aligned}
& X_{t}=A_{1} X_{t-1}+A_{2} X_{t-2}+\ldots+A_{8} X_{t-8}+C \varepsilon_{t}, \ldots . . \text { donde.... } \varepsilon_{t}=\left[\begin{array}{c}
e_{t}^{t} \\
e_{t}^{g} \\
e_{t}^{y}
\end{array}\right] \\
& {\left[\begin{array}{c}
X_{t} \\
X_{t-1} \\
\ldots \\
X_{t-7}
\end{array}\right]=\left[\begin{array}{cccc}
A_{1} & A_{2} & \ldots & A_{8} \\
I & 0 & \ldots & 0 \\
0 & I & \cdots & 0 \\
\ldots & \ldots & \ldots & \ldots \\
0 & \cdots & I & 0
\end{array}\right]\left[\begin{array}{l}
X_{t-1} \\
X_{t-2} \\
\ldots \\
X_{t-8}
\end{array}\right]+\left[\begin{array}{c}
C \\
0
\end{array}\right] \varepsilon_{t}} \\
& \Rightarrow x_{t}=\Phi x_{t-1}+\Theta \varepsilon_{t}
\end{aligned}
$$

donde

$$
x_{t}=\left[\begin{array}{c}
X_{t} \\
X_{t-1} \\
\cdots \\
X_{t-7}
\end{array}\right], \Phi=\left[\begin{array}{cccc}
A_{1} & A_{2} & \ldots & A_{8} \\
I & 0 & \ldots & 0 \\
0 & I & \cdots & 0 \\
\cdots & \cdots & \cdots & \cdots \\
0 & \cdots & I & 0
\end{array}\right], \Theta=\left[\begin{array}{l}
C \\
0
\end{array}\right]
$$

Esta representación del VAR en el espacio AR(1) es muy útil porque nos permite encontrar la función impulso respuesta de forma muy simple. De hecho, la función impulso-respuesta, $\mathrm{IR}_{\mathrm{t}}$,es: 


$$
I R_{t}=\Phi^{t-1} \Theta
$$

Para calcular las desviaciones estándar de las funciones impulso-respuesta, seguimos a Runkle (1987) y utilizamos un procedimiento tipo boostrapping. El procedimiento es correr nuestro VAR, y obtener los errores estimados $\left\{\hat{e}_{1}, \hat{e}_{2}, \ldots, \hat{e}_{T}\right\}$ y los coeficientes estimados $(\hat{\Phi}, \hat{\Theta})$, donde $\mathrm{T}$ es el tamaño de la serie de tiempo. Posteriormente, tomamos una muestra aleatoria de tamaño $\mathrm{T}$ de los errores estimados, donde cada error tiene probabilidad (1/T) de ser elegido, y usamos esta muestra aleatoria de errores para construir una muestra artificial, como la siguiente:

$$
\hat{x}_{t}^{i}=\hat{\Phi} \hat{x}_{t-1}^{i}+\hat{\Theta} u_{t}^{i}
$$

donde $u_{t}^{i}$ es la muestra aleatoria de errores y $\hat{x}_{t}{ }^{i}$ es la muestra artificial construida. A esta muestra artificial se le estima un nuevo VAR y se obtiene una nueva función impulso-respuesta, $I \hat{R}_{t}^{i}$. Este procedimiento se repite 10.000 veces, lo que permite obtener igual número de funciones impulso-respuesta artificiales que se ocupan para calcular la desviación estándar de la función impulso-respuesta del VAR.

\section{Resultados}

El procedimiento descrito en la sección anterior nos permite estimar los efectos de un aumento exógeno del gasto de gobierno e impuestos sobre el PIB.

Los gráficos 1 a 6 muestran la evolución de las variables de mayor interés ante un aumento exógeno (shock positivo) de gasto fiscal y ante un aumento exógeno (shock positivo) de impuestos. Las tres figuras al lado izquierdo muestra los efectos de un shock de gasto, mientras que las figuras al lado derecho muestran el impacto dinámico de un shock de impuestos. Los resultados indican las respuestas en un horizonte de 2 años y medio (10 trimestres). Todas las figuras están construidas en base a intervalos de confianza del $5 \%$.

Los gráficos muestran que un shock de gasto fiscal tiene un efecto negativo en el PIB durante el primer trimestre. El efecto, si bien significativo, tiene una magnitud sólo del orden de $-0.3 \%$. Posteriormente los efectos sobre PIB no son significativos. En el caso de un shock positivo de impuestos, se observa inicialmente también una caída en PIB, que aunque es estadísticamente significativa su magnitud no es económicamente relevante, al caer sólo en $-0.03 \%$ ante el aumento de $1 \%$ en impuestos. Es interesante notar que los efectos de los shocks de impuestos y gastos sobre sí mismos, es decir, sobre impuestos y gastos respectivamente, se hacen no significativos con bastante rapidez. En el caso de impuestos, el efecto aparece significativo sólo dos trimestres, mientras que en el caso de gasto fiscal el efecto es significativo durante un solo trimestre. Esta escasa inercia en el sistema explica el hecho de la poca inercia en los efectos sobre el PIB. 
GRAFICOS 1 A 6

RESPUESTA DE VARIABLES ENDOGENAS A CAMBIOS EXOGENOS DE POLITICA FISCAL
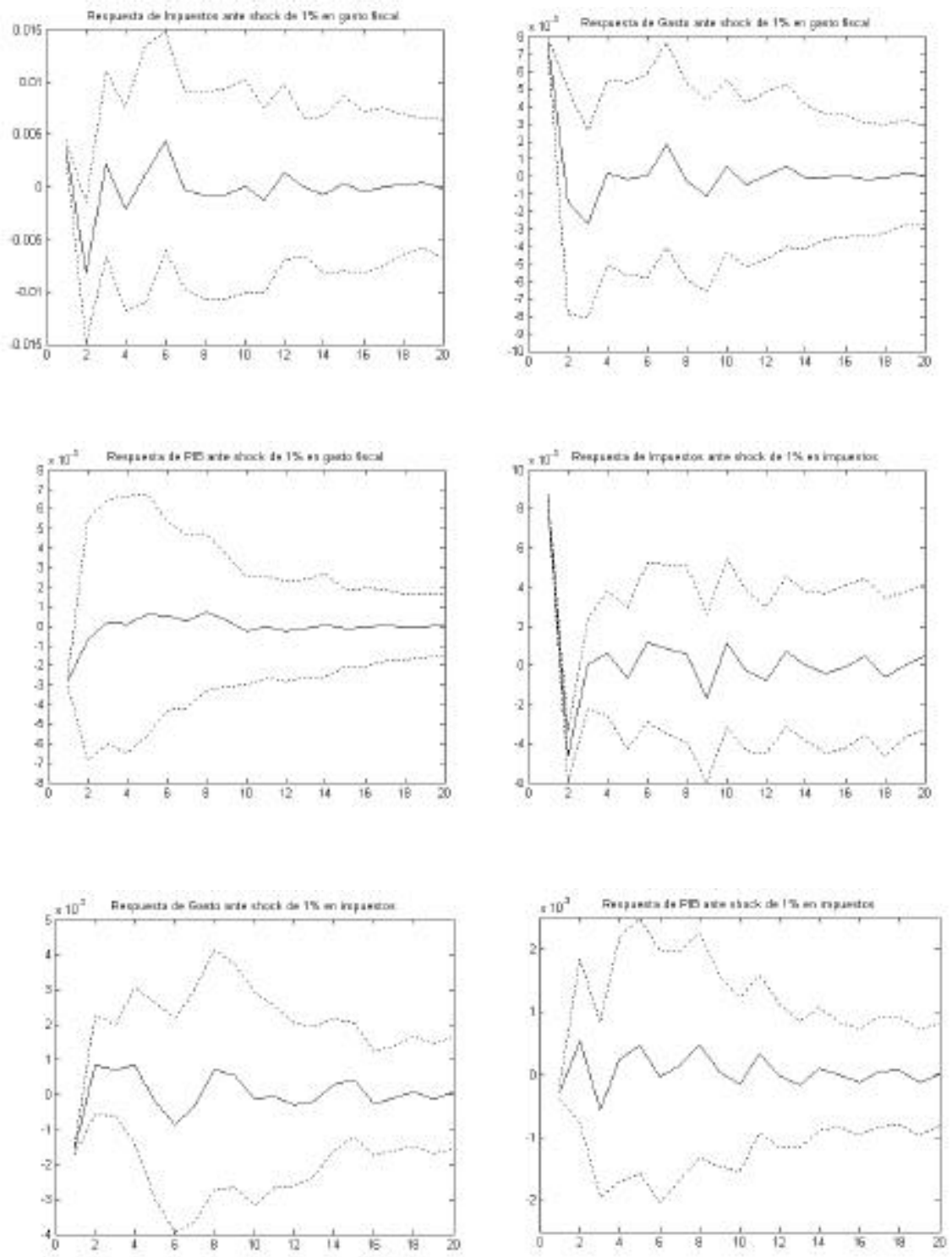
¿Son consistentes los resultados con la evidencia internacional? Al comparar los resultados de este estudio con otros que han utilizado similar técnica econométrica (Blanchard y Perotti 1999 y Perotti 2002) para países desarrollados, se encuentran resultados mixtos. Por un lado, Blanchard y Perotti (1999) encuentran efectos positivos de un shock de gasto fiscal sobre el PIB, lo que es contrario a nuestros resultados. Sin embargo, como puede observarse en el Cuadro 3 que reporta los resultados de Perotti (2002) para países de la OECD a partir de 1980, existen bastantes casos en que un shock positivo de gasto impacta negativamente el PIB. Sin embargo, es importante notar que los efectos en el caso chileno tienen una magnitud considerablemente menor a las obtenidas en los países de la OECD, aunque hay que notar que los shocks de gasto del trabajo de Perotti son mayores. ${ }^{3}$

El Cuadro 4 compara el impacto del aumento en impuesto en los países de la OECD reportados por Perotti (2002) con los resultados obtenidos de nuestras estimaciones. La reacción del PIB observada para el caso chileno es bastante concordante con la evidencia de los países de la OECD, aunque la magnitud del efecto es bastante menor. Nuestros resultados en relación al shock de impuestos son similares a los encontrados por Pierotti (2002) en Canadá y Estados Unidos. ${ }^{4}$

CUADRO 3

IMPACTO DE UN AUMENTO EXOGENO DE 1\% EN GASTO FISCAL SOBRE PIB

\begin{tabular}{|c|c|c|c|c|c|c|c|c|c|c|c|c|}
\hline & 1 Trim. & & 4 Trim. & & 12 Trim. & & 20 Trim. & & Max & & Min & \\
\hline USA & 0.07 & & 0.20 & & -0.53 & & -1.26 & * & 0.49 & (3) & -1.26 & $*(20)$ \\
\hline Alemania Oeste & 0.80 & $*$ & -0.72 & * & -0.86 & & -0.71 & & 0.8 & $*(1)$ & -1.55 & $*(7)$ \\
\hline UK & -0.18 & $*$ & -0.23 & & -1.30 & * & 1.08 & $*$ & -0.01 & (3) & -1.52 & $*(15)$ \\
\hline Canadá & 0.07 & & 0.17 & & -2.23 & $*$ & -2.21 & $*$ & 0.17 & (3) & -2.36 & $*(16)$ \\
\hline Australia & 0.59 & * & 0.47 & * & 0.75 & $*$ & 0.62 & $*$ & 0.79 & $*(14)$ & 0.32 & $*(3)$ \\
\hline CHILE & -0.03 & $*$ & 0.0001 & & -0.0003 & & 0.0001 & & 0.001 & (8) & -0.03 & $*(0)$ \\
\hline
\end{tabular}

Fuente: Perotti (2002) y resultados propios. El cuadro muestra los efectos sobre PIB de un aumento de $1 \%$ en gasto fiscal para el caso de Chile. Para los otros países el shock es de un punto porcentual del PIB. Entre paréntesis, junto a los valores máximos y mínimos, aparecen los trimestres en que ocurren estas respuestas. El símbolo “*” indica que la respuesta es significativa. (es decir, 0 no se encuentra dentro de la zona determinada por los intervalos de dos desviaciones estándar).

${ }^{3}$ Los shocks de ese trabajo son de un punto porcentual del PIB. Si consideramos un país cuyo gasto a PIB es 30\%, el shock de Perotti (2002) es tres veces superior al que nosotros consideramos.

${ }^{4}$ Se aplican también los comentarios de la nota 3. 
Efectos Dinámicos de la Política Fiscal

CUADRO 4

IMPACTO DE UN AUMENTO EXOGENO DE 1\% EN IMPUESTOS SOBRE PIB

\begin{tabular}{|c|c|c|c|c|c|c|c|c|c|c|c|}
\hline & 1 Trim. & & 4 Trim. & & 12 Trim. & & 20 Trim. & Max & & Min & \\
\hline USA & -0.17 & $*$ & 0.37 & $*$ & 0.79 & $*$ & -0.11 & 0.91 & $*(9)$ & -0.17 & $*(1)$ \\
\hline Alemania Oeste & 0.24 & $*$ & -0.49 & $*$ & -0.21 & & -0.32 & 0.24 & $*(1)$ & -6.61 & $*(7)$ \\
\hline UK & 0.18 & $*$ & 0.34 & $*$ & 0.05 & & -0.14 & 0.34 & $*(4)$ & -0.14 & (20) \\
\hline Canadá & -0.12 & $*$ & -0.55 & $*$ & -0.44 & $*$ & -0.09 & -0.09 & (20) & -0.67 & $*(7)$ \\
\hline Australia & 0.41 & $*$ & 0.55 & $*$ & 0.20 & & -0.14 & 0.56 & $*(7)$ & -0.14 & (20) \\
\hline CHILE & -0.003 & $*$ & 0.003 & & -0.0002 & & 0.00 & 0.005 & (2) & -0.005 & (3) \\
\hline
\end{tabular}

Fuente: Perotti (2002) y resultados propios. El cuadro muestra los efectos sobre PIB de un aumento de $1 \%$ en impuestos. Para los otros países el shock es de un punto porcentual del PIB. Entre paréntesis, junto a los valores máximos y mínimos, aparecen los trimestres en que ocurren estas respuestas. El símbolo “*” indica que la respuesta es significativa (es decir, 0 no se encuentra dentro de la zona determinada por los intervalos de dos desviaciones estándar).

\section{RESUMEN E Interpretación DE los Resultados}

Este trabajo reporta evidencia respecto a la dinámica de la política fiscal. Los resultados indican que un shock positivo de gasto fiscal tiene un efecto negativo y significativo sobre el producto sólo durante el primer trimestre; mientras que un shock positivo de impuestos tiene un efecto de impacto negativo, pero de muy baja magnitud, sobre el producto.

Tradicionalmente, se espera un efecto positivo de un aumento de gasto y negativo de un aumento de impuestos, sobre el PIB (este es el resultado más estándar en la literatura). Sin embargo, nuestro trabajo presenta un impacto distinto, lo que puede parecer un resultado no esperado. Para ser justos con la evolución de la literatura, un efecto negativo sobre el producto de un shock positivo de gasto fiscal hoy en día no resulta extraño. De hecho, como concluye el estudio de Perotti, en el período post 1980 los multiplicadores negativos del gasto de gobierno son la norma. Nuestro trabajo aporta más evidencia en ese sentido, ahora desde la perspectiva de una economía pequeña y en desarrollo.

Como en el trabajo no se presenta un modelo analítico para capturar los efectos de la política fiscal, sólo podemos especular respecto de los posibles mecanismos de transmisión que explicarían los resultados. Nuestros resultados son coherentes con lo que se ha denominado el enfoque expectativas de la política fiscal que explica resultados no keynesianos para la política fiscal (Bertola y Drazen 1993, Giavazzi y Pagano 1990 y 1995, Giavazzi, et al. 2000). Un ajuste fiscal puede afectar las expectativas sobre el curso futuro de la política fiscal. En efecto, si el ajuste reduce el gasto fiscal respecto del PIB en forma permanente, se podría anticipar una reducción futura de los impuestos, generándose un incremento en el ingreso permanente y por consiguiente en el consumo privado. Adicionalmente, también podría verse afectada la inversión al aumentar su retorno esperado. Análogamente, aumentos de gasto fiscal podrían tener efectos contractivos sobre la actividad económica, como se reporta en este trabajo. 
Un caso que puede servir como ejemplo es el ajuste fiscal de 1985 que redujo el gasto de gobierno desde un 32,7\% del PIB a un 22,4\% el año $1990^{5}$, junto a una recuperación del crecimiento económico. Si bien las altas tasas de crecimiento observadas podrían explicarse por importantes reformas del período, ${ }^{6}$ el ajuste fiscal constituiría un cambio de régimen, señalando la intención de una disminución permanente de la participación del gasto fiscal en el producto. Esta señal pudo haber sido interpretada como información de una reducción futura de impuestos, como efectivamente aconteció.

¿Cuál de todos los factores que antes enunciamos es el detonante? No tenemos respuesta a esta pregunta. Lo que sí está claro es que se reporta evidencia que puede abrir preguntas para posteriores investigaciones.

\section{REFERENCIAS}

Alesina, A. y R. Perotti (1996), "Reducing Budget Deficits" Swedish Economic Policy Review, 3: 115-134.

Alesina, A. y R. Perotti (1997), "Fiscal Adjustments in OECD Countries: Composition and Macroeconomic Effects", Staff Papers 44 (2): 210-248.

Bertola, G. y A. Drazen (1993), "Trigger Points and Budgets Cuts: Explaining the Effects of Fiscal Austerity," American Economic Review, 11-26, March.

Blanchard, O. y R. Perotti (1999), "An Empirical Characterization of the Dynamic Effects of Changes in Government Spending and Taxes on Output," NBER Working Paper 7269.

Büchi, H. (1993), "Fiscal Policy, Economic Reforms and Private Sector Development: The Chilean Experience." Mimeo.

CEPAL (1993), "La Política Fiscal en Chile: 1985 - 1991,” Serie Política Fiscal 31.

Correa V.; A. Escandón; R. Luengo and J. Venegas (2003), "Empalme de Series Anuales y Trimestrales del PIB," Revista Economía Chilena, Banco Central de Chile. 6, 1.

Fatás, A. y I. Mihov (2001), "The Effects of Fiscal Policy on Consumption and Employment: Theory and Evidence," CEPR Discussion Paper 2760.

Giavazzi, F. y M. Pagano (1990), "Can Severe Fiscal Contractions be Expansionary? Tales of Two Small European Countries," NBER Working Paper 3372 .

Giavazzi, F. y M. Pagano (1995), "Non - Keynesian Effects of Fiscal Policy Changes: International Evidence and the Swedish Experience," NBER Working Paper 5332.

Giavazzi, F.; T. Jappelli, y M. Pagano, (2000), "Searching for Non - Linear Effects of Fiscal Policy: Evidence from Industrial and Developing Countries,” NBER Working Paper 7460.

\footnotetext{
${ }^{5}$ Véanse Büchi (1993) y Larraín y Vergara (2000) para una revisión de la reforma tributaria y ajuste de gastos del gobierno.

${ }^{6}$ Reformas a la salud y previsión, avances en el control de la inflación y la puesta en marcha de novedosas soluciones a la crisis financiera de 1982-83.
} 
Larraín, F. y J. Sachs (2002), Macroeconomía en la Economía Global, Pearson Education.

Larraín, F y R. Vergara (2000), Un Cuarto de Siglo de Reformas Fiscales en Larraín y Vergara (ed.) La Transformación Económica de Chile, CEP.

Marcel, M. et al. (2001), "Balance Estructural: La Base de la Nueva Regla de Política Fiscal Chilena”. Economía Chilena, 4 (3): 5-27.

Marcel, M.; M. Tokman; R. Valdés and P. Benavides (2001), "Balance Estructural del Gobierno Central. Metodología y Estimaciones para Chile: 1987-2000”. Ministerio de Hacienda, Chile.

Perotti, R. (1998), "Fiscal Policy in Good Times and Bad," Columbia University and CEPR.

Perotti, R. (2002), "Estimating the Effects of Fiscal Policy in OECD Countries," ECB Working Paper 168.

Runkle, D. (1987), "Vector Autoregressions and Reality", Journal of Business and Economic Statistics, 5: 437-442.

Tesorería General de la República (varios años) Informe Financiero del Tesoro Público. van Aarle, B.; H. Garretsen and N. Gobbin (2003), "Monetary and Fiscal Policy Transmission in the Euro Area: Evidence from a Structural VAR Analysis". Journal of Economics and Business, 55 (5): 609-638. 